\title{
Perception and Barriers to Prevention of Mother to Child Transmission (PMTCT) of HIV among Pregnant Women Attending Antenatal Clinic in Urban Maternity, Bauchi State, Nigeria
}

\author{
Saba'atu Elizabeth Danladi ${ }^{1}$, Elochukwu Ebunoluwa Ukatu², Osita Samuel Okonkwo ${ }^{3}$, Oruayefe Agatha \\ Upaka $^{4}$, Shakirat Ganiyu Oyindolapo ${ }^{5}$ Iliya Sarki Dongs ${ }^{6 .}$ \\ ${ }^{1}$ International University, Bermenda, Cameroon. Email: sabaatu71@yahoo.com, Mobile: +2348037439790 \\ /+2348028787941
}

${ }^{2}$ Migration Health Assessment Center (MHAC), International Organization for Migration, No. 29 Kaara Street, Ajao Estate, Isolo- Lagos. Email: ukatuelo@gmail.com

${ }^{3}$ Initiative for Improved Population Health Access and Empowerment (iphAE), Plot 13 Ebeano Estate, New Haven, Enugu, Nigeria. Email: osita@iphae.org, www.iphae.org

${ }^{4}$ FHI360, Akwa Ibom State Office, Plot 67, Unit C, Bennett Bassey Street, Ewet Housing, Uyo, Email: oupaka@gmail.com

${ }^{5}$ Department of Obstetrics and Gynaecology, Federal Medical Center, Idi Aba, Abeokuta, Ogun State. Mobile: +2348068426941 Email:shakirat016@gmail.com

${ }^{6}$ International University, Bermenda, Cameroon. Email:iliya_dongs@ yahoo.com, Mobile +234 8033142196

Corresponding author:

Saba'atu Elizabeth Danladi, International University, Bermenda, Cameroon. Email: sabaatu71@yahoo.com

\begin{abstract}
Globally HIV pandemic remains an issue of major concern to the government, health practitioners, policymakers, and researchers. HIV can be transmitted from mother to child either during pregnancy, labor, delivery, or breastfeeding; presently developing countries including Nigeria accord importance to the prevention of mother to child transmission. Despite this effort and commitment to the provision of access to prevention of mother to child transmission of HIV (PMTCT) drugs services in Nigeria, statistics have shown that annual HIV positive births are recorded in larger figures in Nigeria. On this basis, this study aimed to examine the perception of and barriers to prevention of HIV mother to child transmission (PMTCT) among antenatal mothers in urban maternities in Bauchi state, investigating the level of PMTCT services perception, establishing the extent to which PMTCT services are used, and barriers to the use of PMTCT services in the state. The study used a narrative approach and description applying percentages and frequencies using five-point Likert scales to get responses from the respondents. Data were collected and analyzed and the findings reveal a positive perception of prevention of mother to child transmission of HIV and that positive women are ready to take ARV drugs to end the pandemic. It also showed that $90 \%$ of the respondents had good knowledge of HIV transmission mechanisms. The PMTCT program revealed good knowledge of MTCT prevention. We recommend that regular assessment of the PMTCT program should be conducted for program sustainability and scale-up. HIV positive mothers should continue to receive ARV in pregnancy and the postpartum period, reinforce joint action of all stakeholders to reach those women who are positive to achieve the PMTCT national target and religious leaders should preach to their followers to voluntarily have HIV testing to know their status.
\end{abstract}

Keywords: Perception, Barriers, PMTCT, HIV, Antenatal Mothers, Urban Maternity, Bauchi

\section{Introduction}

Prevention of mother-to-child transmission (PMTCT) intervention is recognized globally as an effective method of preventing the spread of the Human Immunodeficiency Virus (HIV) infections. Statistics show that approximately 10 percent of new HIV infections occur through mother-to-child transmission (NACA, 2016, De Cock, et, al., 2000). An HIV positive woman may transmit the disease in utero, during childbirth, or during 
breastfeeding, if the transmission is not checked with treatment. One-third of children living with HIV would die before the age of one year and almost half of them would die in the second year. Globally, the HIV pandemic remains an issue of major public health concern. Statistics from (UNAIDS report) indicate that a total of 35.3 million people are living with HIV; of these, an estimated 2.3 million are newly infected. Out of these, SubSaharan Africa contributes more than two-thirds (69\%) to the global infected population (USAID, 2012). However, children under the age of 15 account for 3.4 million of the global number of those infected, while subSaharan Africa alone contributes $90 \%$ of this burden (USAID, 2012).

To this end, prevention of mother to child transmission is of utmost importance. As the popular saying goes, prevention is better than cure. It is an important way to manage the pandemic and ensure the survival of both mother and child. In support of this, Acharya, Acharya, and Devkota (2018) stated that antiretroviral prophylaxis was given to women during pregnancy starting from 14 weeks+ of pregnancy, delivery, and the postpartum period and lifelong Anti-Retroviral Therapy (ARV), can reduce the risk of dying. Similarly, interventions like prophylaxis for babies started immediately after birth and continued for the first 6 weeks of life can ensure safer delivery practices and safer infant feeding practices (UNICEF, 2009).

Presently, developing countries like Nigeria according to importance to the prevention of mother to child transmission. Antiretroviral drugs for PMTCT reduce the cumulative probability of transmission from as much as 45 percent in the absence of PMTCT to as low as 3 percent (Canning, 2006). Considering the spread of HIV infection among women of the reproductive age, mother-to-child transmission has become a great concern for HIV prevention and health services.

The Nigerian government has devoted resources to fight the HIV pandemic; there is the National Action Committee on AIDs (NACA), an agency of government saddled with the responsibility of managing the HIV statistics and making funds available to fight the scourge. Despite their efforts and commitments to increased access to prevention of mother to child transmission of HIV (PMTCT) drugs services in Nigeria, statistics show that annual HIV positive births of 56,681 (NACA, 2011) are recorded in Nigeria; therefore, much needs to be done. According to NACA, (2011), there is a commitment to expand the coverage of PMTCT services to eliminate the transmission of HIV from mother to child during pregnancy, labor, and breastfeeding. Even in countries with strong PMTCT programs, some level of complacency has been recorded. As such, one of the strategic visions of the World Health Organization is to track program performance and impact on MTCT rates (WHO 2010). From the literature review conducted, a limited number of studies exist in Nigeria on community estimates of HIV disease among pregnant women. However, studies also observed that PMTCT services in urban maternities Bauchi state still fall far short of required standards. This study would fill the gap through identifying perception of prevention of mother to child HIV transmission at urban maternities (PMTCT) in Bauchi state.

Therefore, the study aimed to evaluate the perception of and barriers to prevention of mother to child transmission of HIV (PMTCT) among antenatal mothers in urban maternities in Bauchi state, Nigeria by investigating the level of perception of PMTCT services, establishing the extent to which PMTCT services are used, and constraints to the use of PMTCT services in these facilities the state.

\section{Literature Review}

This study adopted the Tanahashi Model which is also known as the Health Service Measurement Techniques (coverage) (Tanahashi, 1978). The functioning of health services was measured through the understanding and the application of the concept entitled - coverage. A coverage measure is a ratio between the number of people for whom the desired conditions are met and the number of people targeted for a given service. Its evaluation enables the identification of bottlenecks in the operation of the service, to analyze the constraining factors responsible for those bottlenecks, and to select effective measures for service development (Tanahashi, 1978). The model is also based on the premise that the likelihood of engaging in preventive health behavior is influenced by certain beliefs about a given condition. The model asserts that the individual will take preventive health action when they feel susceptible to a certain condition and they feel that contracting the disease has serious consequences compared to the perceived benefits accruing from the same behavior. The AIDS risk reduction model developed in 1990 specifically for AIDS prevention was also used for the study. The model uses constructs from the health belief model to describe the process individuals go through while changing behavior regarding HIV risk. The model identifies three stages involved in reducing risk for HIV transmission. In the first stage, knowledge about HIV transmission and perceived susceptibility to HIV/AIDS influence how women perceive AIDS. The commitment to change is shaped by perceptions of self-efficacy and social norms. In the last stage of health-seeking behavior and social factors affect pregnant women's decision making.

\section{Barriers to the effective utilization of PMTCT service}

According to Abebe, Mengistu, Gete, and Worku (2019), the following factors hinder the effective utilization of PMTCT among HIV positive people.

- Community factors: this includes stigma, discrimination, and limited knowledge of HIV, and so on. 
- Individual and family-related factors: this also includes stigma, discrimination, no partner support, fear of divorce, religious beliefs, lack of family support, lack of time, nutritional problem, lack of awareness, and so on

- Health institution factors: this comprises of cost of the drugs, problems on the provider side, not getting friendly service from health facilities, taking time to get service, and side effects of medications

Furthermore, many barriers to the uptake of PMTCT have also been identified by the world health organization (WHO) which includes:

Knowledge about HIV, MTCT, and PMTCT: People leaving with HIV should be made to be knowledgeable on the disease but this is lacking as the majority of the HIV positive clients are ignorant of it.

Knowledge of HIV status: Antenatal women should know their HIV status, this is vital for pregnant women to access the appropriate treatment and care for themselves and their infants.

HIV stigma, discrimination, and PMTCT: Many researchers have highlighted how HIV-related stigma and discrimination can affect a pregnant woman's decision to enroll in PMTCT programs and interrupt adherence to treatment. Stigma and discrimination are major barriers that can affect the lives of women leaving with HIV

Stigma in healthcare settings: For women living with HIV, experiences of stigma, discrimination, and abuse often occur in health care settings by some health officials when they seek maternal healthcare especially when in labor or during delivery. This might be physical abuse, non-consented clinical care, non-confidential care, non-dignified care, abandonment or denial of care, and detention in facilities.

Hard to reach populations: Restrictive policy environments, stigma, and discrimination in healthcare settings, gender inequality, and economic marginalization undermine access to PMTCT services for women from populations most affected by HIV, such as sex workers and women who use drugs.

Country and clinic resources: In resource-poor settings, shortages of PMTCT staff, interruptions in treatment, and supplies of medical equipment, as well as a shortfall in counselling services, all act as barriers to PMTCT services.

Cultural beliefs and gender dynamics: In many countries, traditional gender roles and cultural beliefs mean that men often make decisions determining women's participation in HIV testing. For example, in many communities in sub-Saharan Africa, pregnancy is viewed as a woman's affair with a man's role primarily to provide financial support, so if a woman is tested positive she might be divorced by the husband and that will be the beginning of her sufferings.

Male involvement: Greater involvement of male partners can also increase retention in PMTCT services and adherence to treatment. Some HIV positive women might have gotten the infections from their male partners because males are likely to engage in extra-marital affairs and refuse to get tested to know their status out of fear of stigma, discrimination, domestic violence, or abandonment by their wives if positive.

\section{Factors Facilitating utilization of PMTCT service}

- Willingness to disclose HIV status which includes individual and family-related issues such as continuous partner support, good support from family, previous history of having HIV free child, feeling healthy, good awareness about HIV transmission);

- Health institution factors which include good health worker support, awareness creation, availability of drugs, discussion during the coffee ceremony with the mother support group, and availing other supplementary support

- Uninterrupted drug supply strengthened mother support group counseling, availing the coffee ceremony budget, and arranging a separate room for counseling.

\section{World Health Organization PMTCT guidelines}

The World Health Organization (WHO) promotes a comprehensive approach to PMTCT programs which includes:

- Preventing new HIV infections among women of reproductive age

- Preventing unintended pregnancies among women living with HIV 
- Preventing HIV transmission from a woman living with HIV to her baby

- Providing appropriate treatment, care, and support to mothers living with HIV and their children and families.

\section{Methodology}

The study used a narrative and approach descriptive approach applying percentages and frequencies using a five-point Likert scale was used to get the needed data from the respondents. However, we adopted the Tanahashi Model which is also known as the Health Service Measurement Techniques (Coverage) (Tanahashi, 1978). This method measures the functioning of health services through the understanding and application of the concept entitled coverage. Hence, the application of a mixed-method narrative and description approach applying both percentages and frequencies using five-point Likert scales for the study.

\section{Results and Discussions}

In this section, the findings based on the study's aims are presented. The presentation utilized a descriptive and narrative approach, applying percentages and frequencies. The study assessed perception on prevention of PMTCT services in urban maternity in Bauchi.

\section{Demographic Distribution of the Respondents}

We present in table I, II, and III background of the respondents which included age, educational level marital status, and occupation.

Table 1: Distribution of Respondents by Age

\begin{tabular}{|l|l|l|}
\hline Age & Frequency & Percentage \\
\hline Below 20 & 12 & 20 \\
\hline $21-35$ & 42 & 70 \\
\hline $36-49$ & 06 & 10 \\
\hline Total & 60 & 100 \\
\hline
\end{tabular}

Source: Field Survey

Table 1 reveals that the number of mothers below the ages 20 constituted $20 \%$ of the respondents while those in the categories of ages 21 to 35 years constituted $70 \% .10 \%$ of the respondents were between the ages of 36 and 49. This reveals that the respondents represent all the categories of pregnant women ranges from the firsttimers to those in near menopause, aged between 15-49 years.

Table 2: Distribution of Respondents by Education

\begin{tabular}{|l|l|l|}
\hline Level of Education & Frequency & Percentage \\
\hline Primary Education & 15 & 25 \\
\hline Secondary Education & 30 & 50 \\
\hline Tertiary Education & 12 & 20 \\
\hline Others & 03 & 05 \\
\hline Total & $\mathbf{6 0}$ & $\mathbf{1 0 0}$ \\
\hline
\end{tabular}

Source: Field Survey

Table 2 above reveals that all the respondents had some level of education, due to the availability and accessibility to schools and learning centers. $25 \%$ of them were primary school graduates, $50 \%$ had secondary education, while $5 \%$ of them had other forms of education. The result shows that those who patronize the services of PMTCT presumably had one form of education or the other.

Table 3: Distribution of Respondents by Marital Status

\begin{tabular}{|l|l|l|}
\hline Marital Status & Frequency & Percentage \\
\hline Single & 12 & 20 \\
\hline Married & 42 & 70 \\
\hline Divorced & 06 & 10 \\
\hline Total & $\mathbf{6 0}$ & $\mathbf{1 0 0}$ \\
\hline
\end{tabular}

Source: Field Survey 
It was observed that $70 \%$ of the respondents were married, $20 \%$ were single, while $10 \%$ of the mothers were divorced. The distribution of the respondents showed that married mothers constituted the majority of those attending the ante-natal clinic.

Table 4: Perception of prevention of PMTCT services among antenatal mothers

\begin{tabular}{|l|l|l|l|l|l|l|}
\hline \multirow{2}{*}{} & Statement & 1 & 2 & 3 & 4 & 5 \\
\cline { 3 - 6 } & & \multicolumn{3}{|l|}{ \% response $\mathrm{n}=60$} \\
\hline 1. & Use of condoms protect against HIV infection & 55 & 35 & 08 & 02 & 00 \\
\hline 2 & Unprotected sex leads to HIV infection & 39 & 32 & 07 & 20 & 02 \\
\hline 3 & HIV can be transmitted through breast milk & 40 & 35 & 08 & 12 & 05 \\
\hline 4 & Abstinence from sex can prevent HIV & 43 & 50 & 00 & 07 & 00 \\
\hline 5 & Mosquito transmit HIV through bites & 00 & 10 & 09 & 35 & 46 \\
\hline 6 & Sharing of eating utensils transmit HIV & 30 & 12 & 28 & 20 & 09 \\
\hline 7 & HIV can be acquired through hands shaking & 14 & 36 & 21 & 17 & 12 \\
\hline
\end{tabular}

$\mathrm{SA}=$ Strongly Agreed 2.A =Agreed 3.U = Undecided 4.D= Disagreed 5. SD= Strongly Disagreed Source:

Field Survey.

The questions were answered using five-point Likert scales. From the table, it revealed that $90 \%$ of the respondents strongly agreed that the use of condoms protects against HIV infection. This finding was confirmed with a study conducted in Ibadan by Adeyi et al., (2006) which reported that $65 \%$ of the respondents identified condoms as a major mode of preventing HIV transmission.

$71 \%$ of the respondents perceived that unprotected sex leads to HIV infection, $27 \%$ of the respondents strongly disagreed or disagreed. $75 \%$ of the respondents strongly agreed or agreed that HIV can be transmitted through breast milk. $88 \%$ of the respondents strongly agreed or agreed that abstinence from sex can prevent HIV infections. $81 \%$ of the respondents affirmed that HIV is not transmitted by mosquito bites in conformity with this finding, 45\% response was found by Adeyi et al., (2006). Also, $42 \%$ of the respondents agreed, while $28 \%$ were undecided that HIV can be transmitted through the sharing of utensils. This was observed as a potential reason for discrimination against HIV patients. $50 \%$ of the respondents strongly agreed or agreed that HIV can be transmitted through a hand shake $21 \%$ were undecided while $29 \%$ disregards respectively. The responses recorded in this study indicate progress with the finding reported by the UNGASS Nigeria (2010) which stated limited knowledge of the drivers of the HIV epidemic in Nigeria. The advancement could be attributed to an increase in HIV awareness campaign through behavior change in the media outlets.

Table 5: Knowledge of MTCT causes

\begin{tabular}{|l|l|l|l|}
\hline & Condition & Yes \% & No \% \\
\hline 1 & Pregnancy & 49 & 51 \\
\hline 2 & Vaginal Delivery & 85 & 15 \\
\hline 3 & Breast feeding & 89 & 11 \\
\hline 4 & Breast feeding as the mother wishes & 03 & 97 \\
\hline 5 & Exclusive Breast feeding for 6 months & 02 & 98 \\
\hline 6 & Infant formula. & 11 & 89 \\
\hline
\end{tabular}

Source: Field Survey

Table 5 above shows that the majority of respondents knew that MTCT can occur during delivery and breastfeeding. However, only $49.0 \%$ knew that this transmission can occur during pregnancy.

In rural Nigeria, more than half of the pregnant women recognized pregnancy as the time during which MTCT occurs. This may show that more public sensitization or more counselling sessions are needed for pregnant women. Over three-quarters of respondents in this study were aware of the use of ARVs during pregnancy as a preventive measure compared to less than a quarter in the Nigerian study (Moses and Peter, 2007). This is a positive finding because knowing that there are ways to prevent the unborn child may help mothers to seek antenatal services early.

The Table also shows $97 \%$ of the respondents were aware that breastfeeding exclusively for six months and breastfeeding increases the chances of mother to child transmission. The majority (89\%) indicated that using infant formula only reduces the risk of MTCT. This is in line with the findings of Ngarina et, al., (2009).

Regarding feeding options for babies by HIV mothers, the findings of this study are similar to that of (WHO) 2007 where it was reported that safer infant feeding practices can significantly reduce post-natal MTCT of HIV infection. In developed countries, avoidance of breast-feeding among HIV mothers has virtually eliminated post-natal HIV transmission in infants (WHO, 2007). 
Table 6: Utilization of PMTCT services among antenatal Mothers

\begin{tabular}{|l|l|l|l|}
\hline \multicolumn{1}{|c|}{ S/NO } & $($ statement n=120) & Yes\% & NO\% \\
\hline A & Use of ARV's & 85 & 15 \\
\hline B & Sensitization about MTCT & 63 & 37 \\
\hline C & Infant feeding & 45 & 55 \\
\hline D & Family planning & 61 & 39 \\
\hline E & VCT & 78 & 22 \\
\hline
\end{tabular}

Source: Field Survey

Table 6 above reveals that the services were the use of ARV (85\%) and VCT (78\%) and family planning which they accessed as they came for Ante Natal Care. The table also indicated that $63 \%$ of the respondents prefer MTCT sensitization while only $45 \%$ prefer infant feeding.

Table 7: Constraints to the use of PMTCT services by antenatal mothers

\begin{tabular}{|l|l|l|l|l|l|l|l|}
\hline \multirow{2}{*}{} & \multicolumn{1}{|c|}{ Barriers } & 1 & 2 & 3 & 4 & 5 \\
\cline { 3 - 7 } & & & \multicolumn{3}{l|}{ Percentage response (n=60) } \\
\hline 1. & Distance/transport cost is a barrier to PMTCT use & 35 & 10 & 20 & 20 & 15 \\
\hline 2 & Traditional beliefs is also a barrier to PMTCT use & 30 & 25 & 10 & 22 & 13 \\
\hline 3 & Fear of involuntary disclosure of status is another barrier & 50 & 28 & 02 & 15 & 05 \\
\hline 4 & Ignorance of HIV transmission is a barrier to PMTCT use & 35 & 45 & 15 & 00 & 05 \\
\hline 5 & Stigmatization & 50 & 22 & 13 & 17 & 03 \\
\hline
\end{tabular}

1. SA= Strongly Agreed 2.A =Agreed 3.U = Undecided 4.D= Disagreed 5. SD = Strongly Disagreed Source: Field Survey

Table 7 shows that the majority of respondents (80\%) strongly agreed or agreed that ignorance of HIV is a major problem. To confirm this, the medical staff declared that after delivery, some of the mothers do trust a positive result as true because they feel healthy. This accounted for their coming for medication after delivery. They did not accept the status themselves and did not want people to know that they were positive. Also, they did not have enough information. Both health workers and other people we spoke with indicated that many people believed that when they felt healthy, they were healthy, and therefore they did not have to use the PMTCT. Both health workers and clients reported that if children tested negative on the first test, many mothers thought that the children did not have to come back for additional testing.

About $45 \%$ of the respondents strongly agreed that the lack of transportation and far distances were two important factors that may have affected the decision to use PMTCT services. This may be a problem for those who come from long distances especially rural dwellers. Distance could be a hindrance to seeking services for those who lived far from their local health facility; living within proximity of the facility was reported as a motivating factor to use the services provided. Table 7 also reveals that fear of involuntary disclosure of HIV status was reported to be a barrier for the uptake of services by $78 \%$ of the respondents. Going to the health facility itself could stir up many questions and community suspicion and may have been a reason why some chose not to attend. One female health worker, stated, that. You may find that people come during closing hours so that others cannot see them, the women are afraid that the family will ask what the medication is for. The recommendation about stopping breastfeeding after six months was mentioned by the clients and was difficult for women to accept. If they had to stop breastfeeding after six months, they were afraid that their in-laws will know that they were HIV positive. She also said, sometimes the in-laws tell their brother and children to leave the woman, and they also spread it to everyone". Confidentiality is extremely important because people are afraid to be exposed before the community.

However, some health workers disclosed patients' HIV status due to a lack of respect for confidentiality. Women were afraid that the doctor will disclose their positive status to others.

Stigmatization was found to be a significant issue. About $72 \%$ of the respondents agreed that stigmatization is a major problem hindering the uptake of PMTCT services. The majority of people did not want others in their community to become aware of their HIV status for fear of involuntary disclosure of HIV status, because they were afraid of being stigmatized. The possibility of being stigmatized by others resulted in some 
people not wanting to know their own HIV status. The fear of being stigmatized and wanting to keep HIV status a secret served as hindering factors for the uptake of PMTCT services. An example of stigma was the common belief that someone who was infected with HIV was engaged in promiscuous behavior and had many sexual partners.

It could also be observed from the table above $55 \%$ of the respondents agreed that the traditional beliefs of clients were hindering the uptake of PMTCT services. When people followed such traditional practices, it hindered the mothers from taking their children for postnatal care in the first days following birth, and thus, it also partly hindered the uptake of PMTCT services. Chinkonde et al. (2009) reported that even though several studies have found that lack of privacy and confidentiality negatively affected patient decision to use certain health services, only a few studies propose suggestions for how to overcome these barriers.

\section{Conclusion and Recommendations}

The transmission of HIV from an HIV-positive mother to her child during pregnancy, labor, delivery, or breastfeeding is called mother-to-child transmission. The global community has committed itself to accelerate progress for the prevention of mother-to-child HIV transmission (PMTCT) through an initiative to eliminate new pediatric HIV infections and improve maternal, newborn, and child survival and health in the context of HIV. Elimination of new HIV infections among children can be achieved through the Prevention of Mother -toChild Transmission (PMTCT). PMTCT is an intervention to ensure that no child is born with HIV and it is an essential step to ensuring an AIDS-free generation. The PMTCT initiative provides drugs, counselling, and psychological support to help mothers safeguard their infants against the virus.

Recent developments suggest that substantial clinical and programmatic advantages can come from adopting a single, universal regimen both to treat HIV-infected pregnant women and to prevent mother-to-child transmission of HIV. This streamlining should maximize PMTCT program performance through better alignment and linkages with antiretroviral therapy (ART) programs at every level of service delivery. One of WHO's two currently recommended PMTCT antiretroviral (ARV) program options, Option B, takes this unified approach. Further evolution - not only provide the same triple ARV drugs to all HIV-infected pregnant women in the antenatal clinic setting but also continuing this therapy for all these women for life.

The importance of the treatment regimen and service delivery includes harmonization with ART programs as well as protection against mother-to-child transmission in future pregnancies. While these benefits need to be evaluated in program settings; systems and support requirements also need careful consideration.

The PMTCT program in urban maternity resulted in high scores in mothers' knowledge of various routes of HIV transmission including oral sex, unprotected sex, multiple sexual relationships, anal sex, and MTCT through breast milk. The findings reveal that perception of prevention on mother to child transmission of HIV is positive and that positive women are ready to take the ARV drugs to end the pandemic but barriers exist and hinder effective utilization of drugs and effective treatment of HIV patients.

Therefore conclude that more needs to be done to eliminate the barriers to achieve better results. This underscores the need to prioritize and target HIV prevention efforts in disproportionately affected communities and ensure that both individual and social determinants of risk are considered in the design and implementation of prevention efforts. Therefore, it is important to counsel pregnant women thoroughly and adequately so they can have this very useful information to prevent mother to child transmission in their present as well as future pregnancies.

The study offers these recommendations for stakeholders involved in the practice of PMTCT.

- Regular assessment of the PMTCT program should be conducted for program sustainability and scaleup.

- Infant feeding counseling should be reinforced and the practices monitored closely in this program as this has been found to contribute to child ill-health and transmission of HIV infection from the mothers to their children.

- HIV positive mothers should continue to receive ARV in pregnancy and postpartum periods according to the current guideline.

- Men involvement is necessary and they need to be encouraged and sensitized from the communities to accompany their partners to the clinic. and attend counseling sessions, this will help equip them both with the necessary information on PMTCT.

- The health education/counseling component of the PMTCT program should be strengthened to fill the revealed knowledge gaps, especially with the transmission routes, high-risk groups, and misconceptions.

- Religious leaders should preach to their followers to voluntarily have HIV testing to know their status. 


\section{Acknowledgment of Support}

This research work has been supported technically by the Geneva Foundation for Medical Education and Research (GFMER), Chemin de Beau-Soleil 12,1206 Geneva - Switzerland. Additional funding support was provided by the African HUB for Community Progress Initiative in Nigeria (AfriHUB), No. 3 Off Judges Quarters, 3RD, GRA, Bauchi, Nigeria. The author also acknowledged the support given by the School of Medicine and Public Health, International University, Bamenda, Cameroun. New life building, Commercial Avenue, P.O. Box 444, Bamenda, Cameroon, Central Africa. However, This research paper contents are solely the responsibility of the authors and the authors highly appreciate both the technical and funding role of the (GFMER) and (AfriHUB)

\section{References}

Abebe, Z.Z. Mengistu, M.Y. Gete, Y.K. \& Worku, A.G. (2019) Factors influencing prevention of mother to child HIV transmission service utilization among HIV positive women in Amhara National Regional State, Ethiopia: A thematic content analysis. CC-BY International License publication.

Adeyi, O. Kanki, P.J. Odutolu, O. \& Idoko, J.A. (2006). AIDS in Nigeria: A nation on The Threshold. Harvard University. Press Harvard series on Population and international health.

Chinkonde, J. R. Johanne, S. \& Francis, M. (2009). The prevention of mother-to-child HIV transmission programme in Lilongwe, Malawi: Why do so many women drop out?. Reproductive Health Matters, 17 (15) 44-56.

De Cock, K. Mary G.F. Eric, M. \& Isabelle, D.V \& Joseph, S.B. (2013). Human Health and HIV pandemic. International journal of health sciences, 4 (5) 89-105.

Devkota, R. Trishna, A. \& Radha, A.(2018) Knowledge regarding prevention of mother to child transmission of hiv/aids among antenatal mothers in Nepal. Journal of college of medical sciences, 14 (1) 1-24.

Ho, D. A. Martha, R. \& Nathan S. (2000). Prevention o Mother-To-Child HIV Transmission In Resource-Poor Countries: Translating Research Into Policy And Practice. Journal of The American Medical Association, 28 (9) 75-82.

Canning, D. (2006). The Economics Of HIV/AIDS In Low-Income Countries: The Case for Prevention. Journal of Economic Perspectives, 20 (3)121-142.

Moses, O. Munir'deen, A. \& Peter, A. (2007). Awareness and Knowledge of MTCT of HIV among pregnant women attending a federal medical center in Nigeria. Journal of National Medical Association, 9 (7) 758-763.

Ngarina, M. Kilewo, C. \& Mpembeni, R. (2009). Acceptance of counseling, voluntary HIV testing and use of prophylactic Nevirapine in labour and immediate postpartum at MNH, Tanzania. Tanzania Medical Journal, 21 (1) 1-5.

NACA. (2011). National Agency for the Control of AIDS (NACA). Prevention of Mother-to-Child Transmission (PMTCT).

NACA. (2016). National Agency for the Control of AIDS (NACA). National Information on HIV and AID (PMTCT)

Tanahashi, T. (1978). Health Services Coverage and its Evaluation. Bulletin of World Health Organization (WHO), 56 (2), Geneva. 295 - 303.

USAIDS. Sub-Saharan Africa regional fact sheet 2012. Retrieved from www.unaids.org/en/media/unaids/contentassets/documents/epidemiology/2012

United Nations Programme on HIV/AIDS. Global report: UNAIDS report on the global AIDS epidemic 2013. Geneva: United Nations Programme on HIV/AIDS.

UNAIDS. (2013). UNAIDS Report on the global AIDS epidemic. Geneva: WHO

World Health Organization. (2009). Call to Action: Towards an HIV-Free and AIDS-Free Generation Prevention of 2005.

WHO. (2010). Millennium Development Goals, United Nations. General Assembly. Special Session, World Health Organization. PMTCT Strategic Vision 2010.

WHO. (2007). HIV and Infant Feeding: New evidence and programmatic experience - Report of technical consultation held on behalf of the Inter-Agency Task Team (IATT) on prevention of HIV infections in pregnant women, Mother and their Infants.

\section{Copyrights}

Copyright for this article is retained by the author(s), with first publication rights granted to the journal. This is an open-access article distributed under the terms and conditions of the Creative Commons Attribution license (http://creativecommons.org/licenses/by/4.0/) 